\title{
Airplane Upset Prevention Research Needs
}

\author{
Judith Bürki-Cohen ${ }^{*}$ and Andrea L. Sparko ${ }^{\dagger}$ \\ USDOT/RITA/Volpe National Transportation Systems Center, Cambridge, MA, 02142
}

This paper, which concludes the Upset Recovery Session convened and chaired by Dennis Crider from the National Transportation Safety Board and the first author at the AIAA Modeling and Simulation Technologies Conference 2008, provides a broad overview of different efforts to prevent airplanes from entering unusual attitudes via Standard Operating Procedures, different types of training and training tools, and airplane-controlprotection and flight-deck technologies. The emphasis will be on providing information that will help determine which research efforts may be most productive in reducing loss of control accidents.

$\begin{array}{ll}\quad \text { Nomenclature } \\ g \quad= & \text { gravity acceleration } \\ V_{m c(a)}= & \text { minimum control speed (air) } \\ V_{1}= & \text { takeoff decision speed; the minimum speed in the takeoff, following a failure of the critical engine, at } \\ & \text { which the pilot can continue the takeoff and achieve the required height above the takeoff surface } \\ & \text { within the takeoff distance }\end{array}$

\section{Loss of Control Continues to be an Issue and May Increase}

66 T oss of control [LOC] is the only type of accident that is not reducing in world-wide statistics," worried the British Civil Aviation Authority's (CAA) Safety Regulation Group in its 2006 Plan. ${ }^{1}$ The Boeing Company’s

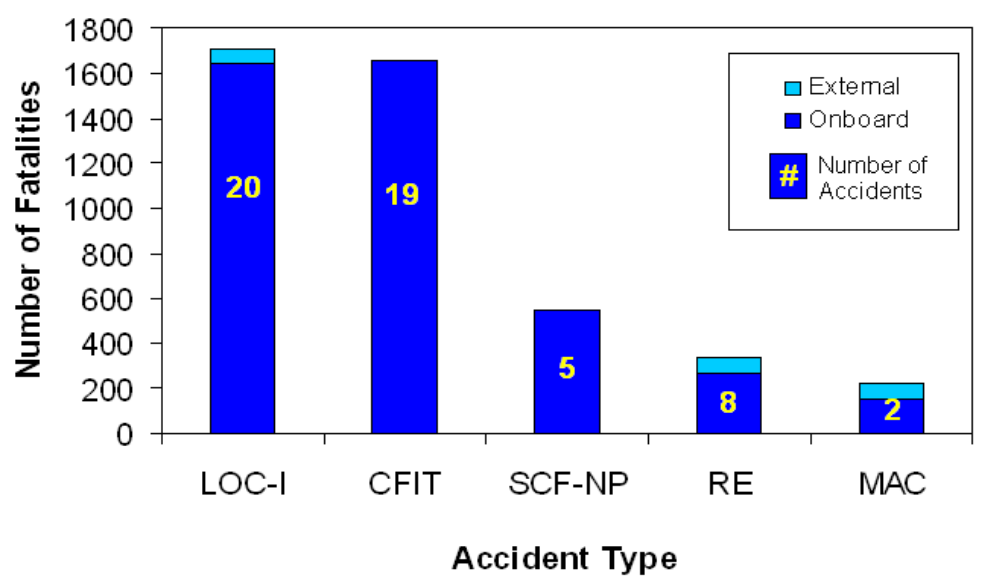

Figure 1. Top 5 fatal accidents, worldwide commercial jet fleet, 1997-2006 (LOC-I: In-flight LOC; SCF-NP: System/Component Failure (non-engine); RE: Runway Excursion; MAC: Midair Collision)
Statistical Summary of Commercial Jet Airplane Accidents $^{2}$ shows that this type of accident has been surpassing the former leader in world-wide accidents, Controlled Flight into Terrain (CFIT) (see Figure 1). ${ }^{\ddagger}$ This is despite universal efforts to improve training methods, training tools, regulatory guidance, airplane protections, and flight-deck warning and advisory systems. LOC accidents may even increase with the advent of Next Generation Air Transportation System (NextGen). For example, decreases in spacing of

\footnotetext{
*Engineering Psychologist, Human Factors Division RTV-4G, 55 Broadway, Member AIAA.

${ }^{\dagger}$ Engineering Psychologist, Human Factors Division RTV-4G, 55 Broadway, Member AIAA.

${ }^{\ddagger}$ Modified from Ref. 2.
} 
aircraft could lead to increases in wake-vortex encounters. In turn, pilots' responses to these may be rendered less effective due to distractions and response delays from NextGen-related automation. Without effective URT or other mitigation, this may lead to increased wake-vortex incidents or even accidents. Other factors that may exacerbate the occurrence of LOC are the increasingly complex mix of airplanes sharing the same airspace. Airplanes are getting larger, smaller, lighter; they will fly faster and higher; short- and vertical-takeoff-and-landing aircraft may compete for slots at the same airports; flight crews are being reduced from once three to, for cargo in "the medium term," one or even zero (Ref. 3, slide 9). An Airbus 380 may encounter a Very Light Jet, an Unmanned Aerial Vehicle, or, dependent on where they will be able to fly, even a supersonic airplane, complicating the issues of spacing and wake vortices. The general interest in the topic of LOC prevention is evidenced by over forty talks addressing LOC from many different perspectives at the 2008 combined AIAA Atmospheric Flight Mechanics; Guidance, Navigation and Control; and Modeling and Simulation Technologies (MST) Conferences. This paper, which is presented last in the Upset Recovery Session convened and chaired by Dennis Crider from the National Transportation Safety Board and the first author at the MST Conference, provides a broad overview of efforts to prevent airplanes from entering unusual attitudes via Standard Operating Procedures (SOP), different types of training and training tools, and airplane-control-protection and flight-deck technologies. The emphasis will be on providing information that will help determine which research efforts may be most productive in reducing LOC accidents. Note that in our discussion, we sometimes stray from the strict definition of airplane upsets, including maneuvers or situations that may eventually lead to upsets.

\section{LOC Prevention: Standard Operating Procedures}

SOPs are the simplest way of preventing LOC, and if every crew at all times regardless of what else goes wrong would heed the mantra "Aviate, Navigate, Communicate," which in the nineties has been modified to include "Manage systems" as a fourth priority, LOC could be prevented in many cases. The Boeing/Airbus "Airplane Upset Recovery Training Aid”" (URT Aid), which will be discussed more thoroughly later in this paper, puts it very simply: "at least one pilot must monitor the aircraft at all times" (p. 2.10).

One example where following these Crew Resource Management (CRM) SOPs and providing the pilots with SOPs for "normal procedures peculiar to [airplane for] routine operations" and for "[e]mergency procedures for foreseeable but unusual situations [that crew may control]," as requested in 14 CFR $\S 25.1585^{5}$ would have prevented a spiral dive into the ground is the May 2005 Fairchild Metro III accident in New Zealand. In this fatal cargo-airplane accident, the already delayed crew asked for all fuel to be put in one tank. When in flight with the autopilot engaged, the crew induced a large side-slip angle to balance the fuel in the unusual gravity-crossflow fuel system typical for this airplane. Once the autopilot reached its control limits it disengaged, but the out-of-the-tactilefeedback-loop crew was unable to regain control. "In addition, both pilots may have been monitoring the fuel gauges," observed the Australian Transport Safety Bureau (ATSB) report (as quoted by Ref. 6, see also Aviation Safety Network* for information on this accident).

By the end of the month, the operator issued a SOP that pilots were to request a balanced fuel load prior to engine start. Two later preventative measures by the operator included the addition of crossflow closure to the Lineup and Approach checklists and of cautions on fuel balancing to the autopilot SOPs. In early 2006, the ATSB recommended to the New Zealand CAA that the Aircraft Flight Manual be amended, in concert with the United States Federal Aviation Administration (U.S. FAA), to include a limitation and caution that the autopilot and yaw damper must be disconnected during in-flight fuel balancing. The CAA commenced this action in May 2006, replying that "however no final date for resolution of this matter can be agreed" (as of July 29, 2008 this safety recommendation was still outstanding). ${ }^{\dagger}$ Similar fuel-balancing events with similar airplanes had been reported in the U.S.

\footnotetext{
*Aviation Safety Network, Swearingen SA.227AC Metro III Accident Description. Retrieved July 30, 2008, from http://aviation-safety.net/database/record.php?id=20050502-0

${ }^{\dagger}$ Transport Accident Investigation Commission, Outstanding Safety Recommendations. Retrieved July 29, 2008, from

http://www.taic.org.nz/OutstandingSafetyRecommendations/tabid/95/Page/0/Mode/Aviation/Recipient/CAA/Imple mentationStatus/Open/Keywords/null/IssuedDateOffset/0/Default.aspx
} 


\section{LOC Prevention: Training}

\section{A. Types of Training}

There are effectively three types of training: academics, airplane, and simulated airplane training. Academic training includes the definition of unusual attitudes and airplane upsets, the psychology of spatial disorientation, the aerodynamics of the airplane, and recovery techniques. This information may be conveyed via regular classroom lectures, audiovisual materials, or Computer-Based Training (CBT). The industry standard is the URT Aid mentioned above and described in more detail in Section III.C.3, "Airplane Upset Recovery Training Aid.” The URT Aid states that academic training will enhance subsequent training in the airplane or in the simulator.

For safety reasons, airplane training is limited to aerobatic airplanes such as the Extra 300L or the Beech Bonanza. It has several disadvantages, though. Not only is there a danger of training accidents, but these types of airplanes also have very different dynamics from transport-category airplanes. The URT Aid specifies that it applies only to swept-wing medium-to-large transport airplanes. The authors do not know of any scientific evidence that demonstrates that there is positive (or negative) transfer of training from an aerobatic to a transport-category airplane (see Section III.F, “Is There Scientific Data on the Effects of Different Types of URT?”). Nevertheless, several private companies are offering such aerobatic-airplane URT. It is also integrated into ab-initio training programs such as those of the German airline Lufthansa and the International Civil Aviation Organization's (ICAO) Multi-crew Pilot License program (MPL), which will be described in Section III.C, "What is the Industry Doing?"

Given the limitations of airplane training such as inappropriate airplane type and danger, it would appear that the ideal URT tool would be a simulator, either a high-level ground-based Full Flight Simulator (FFS) or even better an In-Flight Simulator (IFS). In a ground-based device the instructor has total control of the scenario and can record the scenario and the crew's response to it and use this information for effective debriefing. However, there are limited flight-test data available on upset conditions, and even if perfect data were available, all FFS are severely limited in the fidelity of simulating unusual attitudes and upsets by the limited excursions and bandwidth of the hexapod motion system and its inability to maintain sustained accelerations.

Much attention has thus been given to URT in an IFS, which is a real airplane with real visuals and real motion. Computers drive the handling qualities, the control loaders, and the cockpit displays to simulate those of the simulated airplane. This certainly takes care of the visuals and even to some degree of the motion (within certain margins of safety that can be expanded due to the presence of an expert safety pilot), but the fidelity of the simulated airplane is still limited by the quality and availability of the flight-test data used to program the computers. Also, the inherent danger of being in the air constrains the degree of upsets despite the safety pilot, and some of the control that would exist in an FFS may be lost to the existing meteorological conditions. Finally, the problems of availability and affordability and the time investment make this a viable solution for a select few pilots, but not for those of complete airline fleets. Before proceeding to an examination of what airlines are actually doing, we will summarize the existing and upcoming U.S. FAA regulations.

\section{B. FAA Regulations}

The most recent and thus most applicable regulations with respect to URT are contained in the 14 CFR $\S 60^{7}$ (Part 60) Final Rule, Flight Simulation Training Device Initial and Continuing Qualification and Use. Part 60 became law on May 9th of this year after a six-year approval process involving the convention of an FAA/industry Flight Simulation Device Aviation Rule Making Committee to review the Notice of Proposed Rule Making (NPRM) published on September 25, 2002. It contains a listing of the tasks for which each FFS level is qualified. For unusual attitudes the requirement is to stay "within the normal flight envelope supported by applicable simulation validation data" (p. 26505) [emphasis added in this and following quotation]. The FAA thus rejected the industry's suggestion to substitute "the simulation models" for "applicable simulation validation data," stating that this "would allow an individual to go beyond the flight-test validated flight envelope" and that "[t]his is not an acceptable practice because of the lack of information about aircraft performance and handling beyond those limits” (p. 26479). Tables 1, 2, and 3 show upset-recovery related tasks from Part 60 Appendix A: Qualification Performance Standards for Airplane Full Flight Simulators (p. 26491), Attachments 1 through 3. Note that windshear requirements apply only to turbine-powered airplanes [specifically excluding turbopropeller-powered airplanes (turboprops)] as outlined in 14 CFR §121.358 (Part 121.358, Low-Altitude Windshear System Equipment Requirements), ${ }^{8, \neq}$

\footnotetext{
${ }^{\ddagger}$ Edward Cook, Senior Advisor for Flight Training and Simulation Air Transportation Division, FAA AFS-200 (personal communication, July 28, 2008).
} 
Table 1. Part 60, Appendix A, Attachment 1: General Simulator Requirements

\begin{tabular}{|l|l|r|}
\hline Table & Requirement & FFS Level \\
\hline $\begin{array}{l}\text { A1A. Minimum } \\
\text { Simulator } \\
\text { Requirements }\end{array}$ & Approach to stall, Mach, \& maneuver buffet (motion) & B,C,D \\
\cline { 2 - 3 } & Windshear model & C,D \\
\cline { 2 - 3 } & Airframe \& engine icing for some aircraft; terminal area (model) & C,D \\
\hline \multirow{3}{*}{$\begin{array}{l}\text { A1B. Task vs. } \\
\text { Simulator Level }\end{array}$} & Steep turns & A,B,C,D \\
\cline { 2 - 3 } & Approaches to stalls & A,B,C,D \\
\cline { 2 - 3 } & Unusual attitude recovery within envelope & A,B,C,D \\
\cline { 2 - 3 } & Anti-ice \& de-ice system procedures & A,B,D \\
\hline
\end{tabular}

Table 2. Part 60, Appendix A, Attachment 2: Objective Tests

\begin{tabular}{|l|l|r|}
\hline Table & Requirement/Maneuver & FFS Level \\
\hline \multirow{4}{*}{$\begin{array}{l}\text { A2A. Objective } \\
\text { Tests }\end{array}$} & Handling Qualities & \\
\cline { 2 - 3 } & Stall Characteristics & A,B,C,D \\
\cline { 2 - 3 } & Vmc(a) & A,B,C,D \\
\cline { 2 - 3 } & Spiral stability & A,B,C,D \\
\cline { 2 - 3 } & Longitudinal maneuvering \& static stability & A,B,C,D \\
\cline { 2 - 3 } & Windshear at takeoff \& landing & $\mathrm{D}$ \\
\cline { 2 - 3 } & Motion & $\mathrm{D}$ \\
\cline { 2 - 3 } & Buffet at high air speeds or high Mach & \\
\cline { 2 - 3 } & Buffet at approach to stall & \\
\end{tabular}

Table 3. Part 60, Appendix A, Attachment 3: Simulator Subjective Evaluation

\begin{tabular}{|l|r|}
\hline Requirement/Maneuver & FFS Level \\
\hline Takeoff/landing with windshear & A,B,C,D \\
\hline De-icing/anti-icing & A,B,C,D \\
\hline Cruise & A,B,C,D \\
\hline High Mach number handling (tuck, buffet) \& recovery & A,B,C,D \\
\hline Overspeed warning & A,B,C,D \\
\hline High IAS [indicated air speed] handling & \\
\hline Maneuvers & A,B,C,D \\
\hline $\begin{array}{l}\text { High angle of attack, approach to stalls, stall warning, buffet, and g-break (takeoff, } \\
\text { cruise, approach, landing configuration) }\end{array}$ & A,B,C,D \\
\hline Steep turns & A,B,C,D \\
\hline Motion System Effects & B,C,D \\
\hline Approach to stall buffet &
\end{tabular}

14 CFR § 121 (Part 121, Operating Requirements: Domestic, Flag, And Supplemental Operations) Appendices E (Flight Training Requirements) ${ }^{9}$ and $\mathrm{F}$ (Proficiency Checking Requirements) ${ }^{10}$ contain the oldest regulatory references to upset-related maneuvers. As evident from the use of the terms "visual" and "non-visual" simulators (which roughly correspond to today's Level A FFS with or without visual system), ${ }^{\S}$ these appendices predate the Advanced Simulation Plan published in 1980 as Appendix H. They were written under the assumption that most training and checking is conducted in the airplane, with the exception of unusual attitudes, which can, and windshear, which has to be trained in a simulator. The maneuvers are similar to but less comprehensive than those listed in Part 60 Appendix A, Attachment 1, Table A1B--Table of Tasks vs. Simulator Level. But some of the listings contain more detail, such as steep turns, which are defined as involving a bank angle of 45 degrees and a heading change of 180 to 360 degrees, to be performed in at least a non-visual simulator. Once the revision of Part 121 Subparts N and O, Training Program and Crewmember Qualifications, respectively, will have become effective,

${ }^{\S}$ Edward Cook, Senior Advisor for Flight Training and Simulation Air Transportation Division, FAA AFS-200 (personal communication, July 28, 2008). 
these Appendices, Appendix H, and a new Appendix N will be reserved for Qualification Performance Standards for pilots, engineers, flight attendants, dispatchers, respectively. ${ }^{* *}$

As of this writing, Subpart $\mathrm{N}$ has been cleared by the Office of Management and Budget and is scheduled to be published in the Federal Register as an NPRM by the end of August, followed by a comment period of $30^{11}$ or 120 days. $^{* *}$ It is anticipated that this rule will require the use of Flight Simulation Training Devices (FSTDs) for all flight-crew training. With regard to URT, it is anticipated that the focus will shift to awareness and recognition training from the out-the-window (OTW) view and from the instruments. All URT will be restricted to maneuvers within the validated flight envelope and in the highest level of FFS with the motion turned on. Some training of wake vortex and other atmospheric disturbances may also be included. The URT Aid may also be mentioned.

Additional FAA guidance is contained in FAA Order 8900.1, Flight Standards Information Management System $^{12}$ (FSIMS), dated September 13, 2007, Chapter 19: Training Programs and Airman Qualifications. This order is subordinate to any regulations in 14 CFR and indeed, there are differences between the tasks set forth here and in the Part 60 tables, but Part 60 explains that there should be a difference between the tasks used to assess FSTD characteristics and the tasks for which an FSTD is qualified. For the ground curriculum, FSIMS specifies adverse weather practices and procedures specific to the airplane such as icing, turbulence, windshear, and microburst. The flight training curriculum contains maneuver and procedure tables, mostly similar to the content of Part 121 Appendices E and F, with $\mathrm{V}_{\mathrm{mc}}$ demonstration and recovery, steep turns, approaches to stalls in takeoff and en-route configuration, windshear/microburst, anti-icing and de-icing systems, stall-warning and avoidance devices, and stability-augmentation devices. This document also puts special emphasis on windshear training, which can be done in a Level 7 Flight Training Device (FTD) or any level FFS in accordance with the FAA's "Windshear Training Aid," ${ }^{, 13}$ but windshear training must not be performed in the airplane. A section on LOC accident avoidance training and checking requirements asserts that pilots should be trained in "avoidance and recovery from inadvertent encounters with [Instrument Meteorological Conditions]" (p. 46), "recovery from unusual attitudes" (p. 47), and in addition to training, "the operator should establish an LOC avoidance policy" (p. 47). This document replaces Information Bulletin for Air Transportation 95-15, which contained guidance on URT for MD-11 aircraft based on the 1993 crash of China Eastern Airlines Flight 583 involving a high-altitude upset, and Handbook for Air Transportation 95-10 (Selected Events Training), which advised training for certain URT-related maneuvers.

\section{What is the Industry Doing?}

\section{Airlines}

Most U.S. airlines train upset recovery both in the classroom and in the simulator. ${ }^{14, \dagger+}$ Some incorporate the materials from the URT Aid (see below) in their training and briefing materials, whereas others have generated their own materials. Most use a Level D FFS with the motion turned on. They train fleet-specific scenarios using programmed "hard buttons" or pilot-induced upsets such as excess pitch angle (above 20 degrees) with high and low nose and excess bank angles (above 35 degrees). They perform these maneuvers at high (10,000 feet) and low altitudes, the latter in landing configuration. They may add turbulence for some maneuvers, and some use Windshear Training Aid ${ }^{13}$ maneuvers. They also demonstrate wake vortex and stalls. When asked why they are performing this training in the simulator with motion on, some say that their Principal Operations Inspector requires it.

The German Airline Lufthansa, however, does turn the motion off during their Level D FFS URT for the Initial Type Rating in the Boeing 737 and their Airbus fleets. It emphasizes staying within the validated envelope. It also offers voluntary CBT. URT is offered only during initial type rating." recurrent training, but recurrent pilots are required to undergo simulated windshear training every three months. ${ }^{15}$ During ab-initio training by a company-owned subsidiary in the United States, trainees perform two aerobatic Beech Bonanza URT missions, one each in Instrument and Visual Flight Rules (IFR and VFR). Lufthansa's goal is to prevent pilots from ever getting into an upset condition.

\section{ICAO's Multi-Crew Pilot Licensing}

The ICAO has recently developed an ab-initio training program to prepare candidates for the right seat of an advanced airliner. This MPL also relies on aerobatic training in small airplanes (Ref. 16, Procedures for Air Navigation Services - Training, PANS-TRG, and Ref. 17, pp. 15, 16, 31). MPL training is based on instructional design principles with needs analyses and competence evaluations. An MPL is obtained by successfully completing

\footnotetext{
${ }^{* *}$ Edward Cook, Senior Advisor for Flight Training and Simulation Air Transportation Division, FAA AFS-200 (personal communication, July 28, 2008).

${ }^{+}$Based on interviews conducted with four airlines.

${ }^{\ddagger \#}$ Burkhard Kruse, Deputy Director Crew Training, Lufthansa (personal communication, July 21, 2008).
} 
four phases. CRM, e-learning, part-task training, and classroom instruction are integrated throughout. Phase 1 serves to acquire Core flying skills in the airplane and in a low-level FTD. Phase 2 serves to acquire the Basic skills for multi-crew operations and takes place in the airplane and in a flight and navigation procedure trainer representing at least a generic turbine-powered, multi-engine and multi-crew airplane if not the specific type that will be flown by the candidate. The device needs to have a cross-cockpit-view daylight visual but does not require a motion system. Phases 3 and 4 require increasingly sophisticated FFSs simulating the specific airplane and must incorporate airtraffic-control simulation. URT is incorporated during Basic training in Phase 2, mainly using aerobatic airplanes, and also the non-motion FTD described above. No further flight or FSTD URT occurs in the final two phases.

3. Airplane Upset Recovery Training Aid

Airbus refrains from URT in FSTDs to avoid negative training. This policy is based on the fact that hexapod motion inadequately represents airplane motion especially for unusual attitudes. Moreover, unusual attitudes are "at the limit of the validated flight envelope where few flight test data are available, so the simulator behaviour is based upon extrapolated data" which cannot "guarantee that the simulator behaviour is conform to the aircraft in [unusual attitudes.]" $§ \S$ Instead, Airbus briefs pilots using the Airplane Upset Recovery Training Aid Revision 1, 2004. ${ }^{4}$ The URT Aid was developed by a team of industry and government representatives and signed by Boeing and Airbus. Its recommendations are restricted to swept-wing turbofan airplanes with more than 100 passengers. This implies the potential for negative training if they are misused for other airplane categories. The URT Aid includes two videos, one containing the definition and causes of upsets and the aerodynamic principles that apply during upset situations and the other different recovery techniques. The accompanying document contains a pilot guide with similar information as in the videos, with a set of multiple-choice-test questions for the pilots in an appendix. In addition, it contains an example URT program describing an academic and a simulator training program. Both programs describe objectives and propose syllabi. The simulator training program was designed to maintain the simulator within the mathematical models and data provided by the airplane manufacturer and thus to avoid negative training. For this purpose, the URT Aid advises that simulator limitations, especially with respect to g-load excursions, be explicitly acknowledged, referring to trainees' prior experience with g-load excursions. It also advises that the operator assess their simulators to ensure their ability to support the proposed exercises, e.g., nose high and nose low (low and high speed stalls) exercises. Flight simulator angle-of-attack vs. side-slip graphs for several airplane models are included in an appendix (Airbus 300/310; Boeing 727/37/47/57/77; MD90/11). The graphs give increasingly larger envelopes validated by flight-test, wind-tunnel/analytical, and extrapolated data, respectively. The exercises contain detailed instructions for the instructor including what type of training they are designed for and what types of error to attend to. With its main message to stay within the envelope, the simulator training may be more successful in helping pilots to recognize and prevent upset conditions than to actually recover from a situation that is beyond the normal airplane envelope.

\section{Ground-Simulation Issues}

The previous discussion shows how much the concerns and practices vary across organizations. The URT Aid states that it is a common instructor error to "[allow the] airplane to exceed [the] limits of the simulator...thus causing negative training" (Ref. 4, p. 3.17). FFS URT is reported to have contributed to at least one accident in this century, when an airline's temporary inhibition of roll control to force pilots to use rudder to recover from simulated wake vortices resulted in a pilot using too aggressive rudder inputs in the real world. ${ }^{18}$ The two main problems are the limits of the validated flight envelope and of the hexapod-platform motion systems commonly used by airline and training-center FFS.

\section{Data Envelope}

Although progress has been made in data extrapolation and predictive mathematical modeling, the non-linear parts of the envelope are still providing a major stumbling block. Other methods to procure the data, such as wind tunnel experiments, use of subscale aircraft, and using accident data have been slow and controversial. Some, including potential funding sources, have lost faith in the ability of such efforts to expand the flight envelope with sufficient confidence. The number of papers addressing the data issue in the combined AIAA conferences is encouraging, however. We are referring to these papers for further information on this issue (see Section I, "Loss of Control Continues to be an Issue and May Increase").

\section{Motion Fidelity}

Studies conducted at the Volpe National Transportation Systems Center (Volpe Center) investigated the effect of hexapod motion on training maneuvers within the validated envelope. The motivation for this research was the

\footnotetext{
${ }^{\S \S}$ Laurent Denaux, Training Devices Qualification Manager, Airbus Training (personal communication, June 30, 2006).
} 
requirement that all airlines operate under the same safety rules (One Level of Safety Initiative) ${ }^{19}$ This requirement implies that all airlines have affordable access to the benefits of simulators for training their pilots. Therefore, the FAA needed scientific evidence that the required simulator-motion cues are both sufficient and necessary to improve transfer of pilot behavior and performance between the simulator and the airplane. Previous studies had found no effect of motion on transfer, but may lack statistical power due to insufficient control over the experimental conditions when testing transfer to the airplane. There may also have been too much inter-pilot variability with an insufficient number of pilots in each group to wash it out. Finally, these studies used equipment that today is no longer state of the art.

The FAA/Volpe studies compared two groups of pilots trained in an FAA-qualified Level C or D FFS in a quasitransfer design: one group was trained in the simulator with the motion on and one group was trained in the same simulator with the motion off. Both groups were then tested in the simulator with the motion on (as a stand-in for the airplane) to see if differences in performance and/or behavior were attributable to the presence of motion during training. These studies were carefully designed to ensure the ability to find an effect of motion by: 1) avoiding spurious effects so that the only difference between groups was the presence or absence of physical motion cues; 2) testing large samples of pilots to achieve the statistical power to find an effect; and 3) training diagnostic maneuvers. Extensive data on pilot-vehicle performance and behavior were collected from the simulator, and all participants (pilots flying, pilots not-flying, and instructors) filled out detailed questionnaires on their perception of pilots' actions and workload, the simulator, and their own comfort in the simulator. The maneuvers flown in these studies were deemed most diagnostic of a need for motion by subject-matter experts and the literature; that is, in these maneuvers the motion cues are presumed to give an early warning of outside disturbances such as weather and engine failures. According to some theories, disturbance maneuvers should be perceived more slowly from the visual cues provided by the OTW view and by the instruments than from motion cues. No such advantage is derived from airplane motion cues during upset maneuvers, in fact, for such maneuvers physical motion cues can be misleading. This is because they are perceived by mechanical receptors in our ears and skin, which quickly adapt to constant stimulation and stop firing. Pilots may thus lose the perception that they are in a constant turn, or worse, when the turn rate decelerates, perceive a turn onset in the opposite direction. This may induce pilots to apply the opposite control inputs from those required, leading to excess bank and eventually to a graveyard spiral.

Three FAA/Volpe Center studies found no operationally relevant effects on transfer, neither in the objective data nor in the questionnaires. Ref. 20 investigated the effect of the motion of an FAA-qualified Level C simulator on recognizing and responding to engine failures requiring rejected and continued takeoffs. The study was conducted during recurrent training and evaluation of captains of a 29-seat turboprop. In addition to finding no effect of motion, the study showed that the lateral acceleration of the simulator, arguably the most important motion cue used to recognize engine failures at takeoff, was underrepresented. Subsequently, data from eight other Level C and D simulators was collected and compared to the experiment simulator, revealing that the lateral acceleration of the experiment simulator was actually quite typical. To examine whether stricter regulation of motion cues would lead to operationally relevant effects of motion, a second study ${ }^{21}$ was conducted with NASA, enhancing the motion and adding, among other maneuvers, a hand-flown engine-out Precision Instrument Approach and landing (PIA) with severe weather. The motion system of the Level D B747-400 simulator was tuned to improve translational motion (mainly sway), but that was not possible without trading off rotation (mainly yaw) (see Figure 2). Results of this study did reveal a small effect of motion during training: the experienced pilots in this simulator did use the motion as an alerting cue, and responded slightly faster to engine failures at $\mathrm{V}_{1}$ during training with motion than a similar group of pilots that had to rely on visual cues only. However, this reaction-time advantage did not manifest itself in higher flight precision. Most importantly, the effect did not transfer, once all pilots were provided with motion cues, they all responded equally fast. A third study ${ }^{22}$ which investigated initial training in a Level D B717-200 confirmed the results of Ref. 21, with the exception of a small advantage of being trained without motion on horizontal directional control and general control steadiness found in Ref. 21 for the engine-out PIA. Interestingly, these latter effects did transfer to the simulator with motion. 


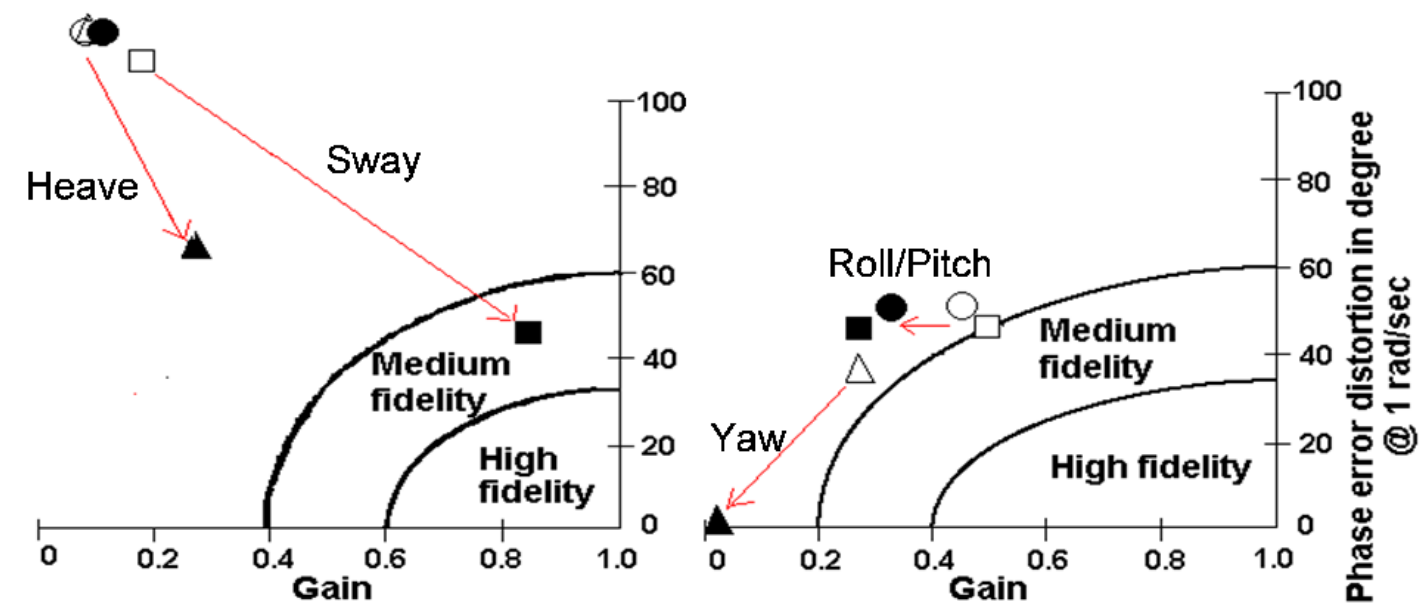

Figure 2. Enhanced translation trading off rotation

These studies were the first studies with demonstrated statistical power to find an effect of motion and showed no benefit of simulator motion for training maneuvers even within the flight-test-data validated flight envelope. This suggests that physical motion cues may not be necessary for successful training and evaluation of air-transport pilots. Despite this evidence and the fact that there exists no scientific confirmation that hexapod motion contributes to transfer, ${ }^{20,21,22,23,24,25}$ a regulatory motion requirement still exists. This is partly attributable to the face value of motion: the airplane moves, so the simulator should move. Also, pilots have been successfully trained with motion for 28 years, which suggests that motion certainly does not impede performance. But, if hexapod motion is insufficient to adequately simulate maneuvers within the normal flight envelope so that motion cues improve training and evaluation, it is questionable whether it will help for upset maneuvers that are outside of the normal envelope. This argument stands also in response to a contention that pilots may need to learn how to ignore their potential misinterpretations of motion cues and to fly by the instruments instead. The limitations of hexapod motion systems and of the available data are insufficient even for that purpose. Putting the data issue aside, however, a few advanced motion systems have been developed that may be able to overcome some of the limitations of hexapods. However, these systems still suffer from the same lack of flight data and are likely beyond the financial and/or physical range of most operators.

\section{E. Advanced Motion Systems}

1. Vertical Motion Simulator

The Vertical Motion Simulator (VMS) at NASA Ames Research Center has the largest motion system in the world. The motion platform can move in all six degrees of freedom (DOF). The simulator cab is mounted on lateral and longitudinal carriages that move up to 40 feet and 8 feet, respectively. They sit on a vertical platform that can provide up to 60 feet of vertical motion. The vertical platform can accelerate up to $22 \mathrm{ft} / \mathrm{s}^{2}$ (almost $0.75 \mathrm{~g}$ ). The cab can also be mounted 90 degrees from its usual position, so that the longitudinal carriage can be used for lateral movement, and the lateral carriage for longitudinal movement. Yaw is controlled by a rotating center and pitch and roll are controlled by actuators. ${ }^{* * *}$ The motion provided by the VMS is capable of realistic g-loading for maneuvers within the normal envelope ${ }^{26}$ including during takeoff and landing, but the maximum loading of the VMS is still not near the maximum design loads of typical civilian (4g) or military (9g) aircraft. ${ }^{27}$

\section{Centrifuges}

Centrifuge trainers provide sustained $\mathrm{g}$ and can be used to train increased g-tolerance and recovery from high-g and spatial disorientation situations. Advanced systems can provide sustained and simultaneous +/-360 degreemotion cues in pitch, roll, yaw and planetary DOFs. The latter DOF is used to provide some translational motion cueing as well. One company advertises g-values as high as 2.5 even for translational motion. Such simulators are

\footnotetext{
${ }^{* * *}$ Vertical Motion Simulator Overview. Retrieved July 31, 2008, from http://www.simlabs.arc.nasa.gov/vms/vms.html
} 
marketed to train pilots to "rely on their flight instruments and maintain control while ignoring the false information that their senses of orientation are providing."

\section{Desdemona}

Desdemona was developed by AMST Systemtechnik (Austria) and is housed at TNO Human Factors in The Netherlands. This simulator can provide sustained $g$ without the angular accelerations experienced in centrifuges during g-onset. Desdemona's fully-gimbaled cockpit provides two meters of yaw, pitch, roll, and heave in 360 degrees, and is mounted on an eight-meter longitudinal track which is rotated around the vertical axis. The track can rotate as a centrifuge at up to $3 g .{ }^{\mathrm{f}}$

\section{F. Is There Scientific Data on the Effects of Different Types of URT?}

The only published study that we know of addressing this question is described in detail in Valerie Gawron's 2002 report “Airplane Upset Training Evaluation Report."28 This study selected five groups of eight pilots each: one

Table 4. Airplane Upset Training Evaluation pilot groups

\begin{tabular}{|c|c|c|}
\hline Group (N) & Beech Bonanza & URT \\
\hline $1(8)$ & No & No \\
\hline $2(8)$ & Yes & No \\
\hline $3(8)$ & No & FFS \\
\hline $4(8)$ & Yes & FFS \\
\hline $5(8)$ & N & IFS \\
\hline
\end{tabular}
group each with no URT (the control group), aerobatic URT only, FFS URT only, both aerobatic and FFS URT, or IFS only. The Veridian Variable Stability Learjet IFS "replicate[d] the handling characteristics of commercial aircraft and the flight deck 'environment'” (p. 28) of a generic wing-mounted twin-engine, mid-size jet transport (see Table 4 for summary of pilot groups and URT regime).

All pilots were recent hires of 27 different airlines in their probationary year and none had prior military experience. Only the IFS URT was provided in the course of the study (11 exercises ${ }^{\S \S}$ preceded by four hours of ground school). Therefore, Groups 3 and 4, which required FFS URT, were recruited from American, Delta, and United, which provide such training. Groups 2 and 4 were made up of pilots that had "relatively recent aerobatic experience...defined as at least six-hours of training completing [various aerobatic maneuvers]" (p. 35). All non-IFS groups were exposed to an approximately 45-minute familiarization flight. Alas, with only eight pilots in each group and minimal control over prior experience and the non-IFS training variables, no differences were found between the five groups during their one-hour evaluation flight in the IFS. It does not speak well for the effectiveness of the IFS training, however, that

Table 5. FAA/FedEx/Calspan study groups and phases

\begin{tabular}{|c|c|c|}
\hline Phase & “Trained Group” (N=10) & “Pseudo Group" $(\mathrm{N}=10)$ \\
\hline 1 & FFS Upset Recovery Evaluation & \\
\hline 2 & Academics & \\
\hline 3 & FFS Upset Recovery Evaluation & \\
\hline 4 & FFS URT & FFS Pseudo Training \\
\hline 5 & FFS Upset Recovery Evaluation & \\
\hline 6 & IFS Upset Recovery Evaluation & \\
\hline $7-12$ & 6 pooled phases including aerobatic t & ning, IFS training, etc. \\
\hline
\end{tabular}
received training as recently as the previous day did not outperform the four other groups. The author's conclusion boils down to "for some scenarios, clearly, training works” (p. xvi), however, this statement is based on the recovery of 39 of 40 pilots from windshear upset and "[a]ll 40" pilots' own attribution of their success to prior training (p. xvi). At least 8 of the 40 had had no

${ }^{\dagger+\dagger}$ GYROLAB® Spatial Disorientation Trainers. Retrieved July 31, 2008, from http://www.etcaircrewtraining.com/ats_gyrolab.php.

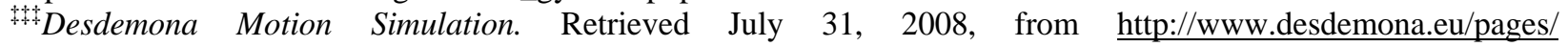
frames desdemona.htm.

${ }^{\$ \S \S}$ No time is given for the IFS training flight, but the statement that "the familiarization flight equalized the familiarity of [the non-IFS groups]" gives the impression that it must have been of similar duration to the familiarization flight, i.e., 45 minutes. 
prior URT, one did not recover, and for the remaining 31 there was no baseline against which to evaluate their performance. However, the study does provide some interesting insight on pilots' recovery strategies and URT needs.

A second as of yet unpublished FAA/FedEx/Calspan study is scheduled to be presented by Brian Ward, FedEx's Managing Director Flight Training, Air Operations Division, at the Flight Safety Foundation's $61^{\text {st }}$ Annual International Air Safety Seminar (IASS), October 27-30, 2008, in Honolulu, Hawaii. The first author attended a preliminary briefing to the FAA given by Calspan's Jim Priest at Fedex headquarters on February 7, 2008. The study trained and tested two groups as shown in Table 5. Phase 4 was the only phase where two groups underwent different treatment; and this for no more than an hour including simulator setup. The "Pseudo Group" in Phase 4 received training equivalent to SPOT (Special Purpose Operational Training) without any unusual attitudes in the FFS, whereas the "Trained Group" did receive URT in the FFS. The preliminary data presented did indicate that the recovery-score improvement of the "Pseudo Group" between the FFS upset-recovery evaluation in Phase 5 and the IFS upset-recovery evaluation in Phase 6 was higher than the one of the "Trained Group" (as determined by two independent raters grading the FFS evaluation and two additional raters evaluating videos of pilots' performance). However, without statistics and knowing the differences between the two groups during each phase no conclusions can be drawn. Too many effects such as inherent differences between and within the groups, or one group having reached a performance ceiling earlier than the other, may account for this difference rather than the short period of differential training in Phase 4. It will be interesting to see what the final and complete results for this study show.

\section{What Else Can Be Done?}

Given that the constraints on URT may limit its success in LOC prevention, alternative systems that prevent upsets or assist pilots in recovery have been developed. Such systems include flight-envelope protection, assisted recovery, and warning systems [e.g., stall warning, weather detection and forecasting, attitude displays, enhanced ground proximity warning system (EGPWS), and synthetic vision systems (SVS)]. These will be discussed in more detail in the following sections.

\section{A. Flight-Envelope Protection}

The prerequisite for flight-envelope protection systems is a fly-by-wire (FBW) system. In the FBW, the pilot's inputs are sent to a computer which then commands the flight controls (there is no direct link between the pilot and the controls). FBW systems have existed for 30 years $^{29}$ but are currently only available in advanced jets such as Airbus models, B777 and 787, Embraer 170/190, and the Falcon 7X. ${ }^{30}$

Flight-envelope protection systems vary, but all offer some level of automation to keep the airplane within safe operating limits. The safe flight envelope is defined by speed, attitude, and angle of attack (AOA), and depends on airplane and environmental factors. It also makes assumptions on pilots' perceptual and aviator skills. Envelopeprotection designers choose how to limit the envelope based on these dependent and mitigating factors, and systems will vary in their degree of automation and system responses. ${ }^{31}$

Soft protections, such as those used in Boeing models, incorporate voluntary pilot-in-the-loop limit protection. These systems inform the pilot when the envelope limit is approaching by using aural and visual warnings or by increasing control feel (stick forces). If necessary, the pilot can choose to override the system and allow the airplane to enter a stall, over-bank, etc. ${ }^{32}$ The Airbus family incorporates hard protections, which are autonomous. These systems do not allow the airplane to be stalled, over-banked, etc., regardless of crew intentions. ${ }^{32}$ Soft systems leave the ultimate authority with the flight-deck crew, while hard systems are more concerned with the integrity of the aircraft. $^{31}$

Research on flight-envelope protections continues. Honeywell is developing an "assisted-recovery" feature which may one day be incorporated into Airbus models. This system is similar to the auto-pull-up concept, where the automatic flight-control system (AFCS) automatically performs the pull-up maneuver at the latest possible moment in response to alerts from the Enhanced Ground Proximity Warning System (EGPWS). ${ }^{33,34}$

NASA is developing a "refuse-to-crash" technology, or an Intelligent Flight Control System, that uses an onboard neural network and control algorithms to identify in-flight problems and adapt aircraft control to maintain optimal flight performance. ${ }^{35, * * * *}$ One such project is Integrated Resilient Aircraft Control (IRAC), which aims to

\footnotetext{
NASA Dryden Fact Sheet - Intelligent Flight Control System. Retrieved August 6, 2008, from http://www.nasa.gov/centers/dryden/news/FactSheets/FS-076-DFRC.html
} 
improve integrated adaptive controls for aircraft to ensure "stability, maneuverability, and safe landing in the presence of adverse conditions" (Ref. 36, p.1).

\section{B. Warning and Advisory System Considerations}

From a human factors perspective, warnings and advisories must: a) be appropriately compelling, i.e., not compete with more urgent information (e.g., obstacles out-the-window); b) be intuitive, easily interpretable, unambiguous, and complete; and c) lead only to desired actions. A major consideration for warning systems is that they must support effective task management and prevent overload within and across sensory modalities. With all of the information displayed on the flight deck, both the visual (head-up or head-down displays) and auditory sensoryinput channels are very heavily taxed. More capacity remains in the tactile domain (control-force feedback and stick shaker), where seat-cushion activation may still be an option. For ultimate emergencies, even smell could be employed, which however has the disadvantage of lingering. The reason for the predominant use of the visual and auditory channel is the density of information that can be conveyed, including the degree of urgency. One disadvantage of visual information is that pilots have to directly look at it. That's why EGPWS' "bank angle" warning, which could be expanded to include the desired action, is given aurally. In summary, in order to be effective, a system must enable pilots to appropriately focus their attention on the information needed for the most important actions (weather displays; aircraft status, including systems, attitude, or vertical situation).

\section{Weather Displays}

External and in-flight weather-forecasting displays are available to pilots to see current and anticipated weather conditions, including precipitation type, lightening, cloud-top information, winds, and temperatures. ${ }^{\dagger+t+}$ Most of these displays are updated every few minutes.

According to Ref. 37, all commercial aircraft are required by the FAA to have on-board windshear detection systems. Some have reactive systems, which determine the presence of windshear from aircraft instruments and report the windshear to the pilot only after the pilot has encountered it. Other, predictive, forward-looking detection systems give pilots advanced warning of windshear with enough time to decide whether to enter windshear (and time to choose the proper maneuver) or to avoid it. Most of these are laser or radar systems which send waves ahead of the aircraft to detect various windshear-related particles and return a signal to the aircraft. NASA Langley, which pioneered the development and evaluation of these systems, reported that this technology typically provides between 20 and 40 seconds of advanced windshear warning (NASA determined a need for 10 to 40 seconds). The only problem with radar systems is that they not only look ahead, but they also look down, picking up clutter on the ground (e.g., cars). Nevertheless, predictive windshear detection systems have proved to be effective--the number of

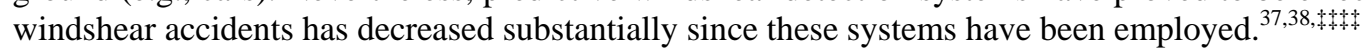

In-flight icing detection systems are also used to guide pilots away from areas where potential icing could occur. Since December, 2006, a system called the Current Icing Potential (CIP), which was formerly available to meteorologists only, was made available to pilots during flight. This system gives pilots icing updates in-flight using plots and maps streamed directly to the cockpit and updated every hour. Information for in-flight CIP is gathered from surface observations, weather models, satellite and radar data, and pilot reports. ${ }^{39}$

2. Aircraft Status Displays

Several displays exist to notify pilots of the orientation of the aircraft. This includes systems that monitor and present information about the control surfaces and operating system (e.g., stall warning) and others that present the airplane's attitude or vertical situation.

The proper display of attitude information is crucial not only to unusual-attitude recognition, but also to determine which action to take to recover. Attitude displays can be head-up or head-down, and there are advantages and disadvantages to both. Head-down displays (HDD) do not overlay the OTW view, yet pilots may need outside visual cues to better understand their orientation. Head-up displays (HUD) can be more quickly interpreted and some have an automatic declutter feature to clear the OTW view, but HUDs typically have no color or texture to distinguish upright from inverted flight and they may have a truncated pitch scale (due to limited FOV), which may move too fast for pilots to recognize pitch direction. ${ }^{40,41}$ Pilots could be trained to use the HDD instead of the HUD during unusual attitudes, however pilots may have to re-adjust to using the head-down indicators after switching from the HUD. ${ }^{40}$

Vertical situation displays (VSD) show an intuitive graphic of the plane's descent profile and the surrounding terrain. These can be useful in determining orientation in the air, but also decrease mental workload so that pilots

\footnotetext{
${ }^{++\dagger \dagger}$ WSI website. Retrieved July 31, 2008, from http://www.wsi.com/

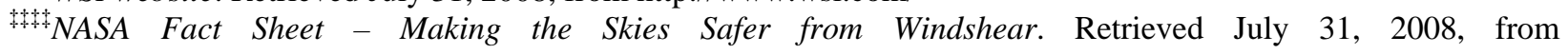
http://oea.larc.nasa.gov/PAIS/Windshear.html
} 
can be better prepared to encounter potential LOC. Without VSDs, pilots must integrate information from a variety of sources (e.g., altitude readouts, vertical speed indicator, GPWS, terrain depiction systems, and navigation information from the flight management computer and navigation charts) in order to form a mental image of vertical position. ${ }^{42}$

3. Synthetic Vision Systems

Synthetic Vision System (SVS) are next-generation flight-deck systems that present computer-generated images of the aircraft's position with respect to terrain. In low-visibility situations, a pilot can glance at the SVS and see what is ahead, as if he or she were flying in the daytime with clear-skies under VFR conditions. SVS combine HUD's altitude, heading, speed with traffic, terrain, and highway in the sky so that pilots can know exactly where the aircraft is and what it is doing at any given moment. With these systems, pilots can reduce multiple instrument cross-checks, thereby saving time, decreasing workload, and increasing situational awareness. SVS reduce the effort of finding crucial data, require little to no interpretation to convert the data into something meaningful, and require just one glance to view status changes so that there is no need to compare the data on the display to what was previously there. ${ }^{43}$ In summary, by presenting a realistic representation of the OTW, SVS provides a more compelling indication of attitude than an ordinary attitude indicator, thus reducing the possibility of pilot disorientation.

\section{Airplane Upset Prevention Research Needs}

LOC is the main cause behind fatal airplane accidents today (see Figure 1). There is reason to believe that this problem may increase in the near future. In addition to SOPs, there are three main approaches addressing this problem, namely, pilot training, airplane-control technologies (flight-envelope protection, automated recoveries), and flight-deck display technologies (situational awareness, warning, and advisory displays).

The main issues in flight-deck display technologies include first and foremost the accuracy of the data (airplane status, weather forecasts, terrain-data bases). Almost equally important to the success in preventing upsets of such systems, however, is the quality of human-factors engineering invested into their design. Although there is still much room for progress in these areas, flight-deck displays may be the least controversial LOC prevention approach. Few would question the success of EGPWS in significantly reducing the risk of CFIT-by 50 percent in Western Europe and North America compared to 1991, "making this one of the biggest success stories in the history of aviation," according to the manufacturer. ${ }^{\S \S \S \S}$ It seems reasonable that similar annunciations, together with advice on how to recover, would be equally successful in LOC prevention. Others, however, would like to rely entirely on airplane-control technologies, such as the head of flight and cabin crew training at Airbus Captain Michel Landrin, who maintains that for Airbus airplanes, thanks to the flight-envelope protection associated with the FBW system, the probability of entering an upset situation is so remote that advanced flight-deck warning and advisory systems are not needed and might even result in a conflict between pilot and automation on who has control of the airplane. $^{* * * * * *}$

This is the main issue in airplane-control technologies: who is in command of the airplane, the pilot or the automated systems? The United States' Air Line Pilots Association's (ALPA) stance is that pilot authority should not be limited, and that the pilot should have the ultimate power to override the system to attain full aerodynamic performance if necessary. ALPA also warns that trying to design-out pilot error with automation may inadvertently replace pilot error with design error. ${ }^{32}$ Any automated airplane-control solutions will remain unpopular as long as pilots remain reluctant to relinquish any of their command to automation. Pilots still mistrust the reliability of envelope protections based on past automation mishaps. The same may be true for solutions that will autonomously return the airplane to a normal attitude, such as assisted recoveries, even if they inform the pilot on what is happening. Until envelope protection and autonomous correction of attitudes have been shown to be reliable in all situations, they will continue to have their detractors.

The main issue in pilot training is to determine the medium, or suite of media, that will result in the highest positive transfer of training (and of course avoidance of any negative transfer--primum non nocere). The available media include academic (classroom, CBT, video, e-learning), simulation (part-task trainer, FTD, FFS, IFS), or aerobatic airplane. The training success of these media is intimately linked to the quality of the information provided. For simulation devices and aerobatic airplanes this refers to how faithfully they represent the target

§§§ Enhanced Ground Proximity Warning System (EGPWS). Retrieved August 01, 2008, from http://www51.honeywell.com/aero/technology/key-technologies2/avionics3/egpws.html?c=23

${ }_{* * * * *}$ Captain Michel Landrin, Head of Flight and Cabin Crew Training, Airbus (personal communication, May 25, 2008). 
airplane with regard to the visual, vestibular, proprioceptive (tactile), and auditory stimulation of the pilot. For simulation devices, the level of fidelity of these cues is constrained on the one hand, by the technical capabilities of the device, and on the other hand by the availability of data either collected or validated in flight. Although the most heavily regulated and the most widely practiced prevention measure, training may also be the most controversial remedy for LOC. Academic URT only, mainly aerobatic, FFS with motion, FFS without, IFS, real URT or recognition, prevention and familiarization only...all these options and no scientific data that we know of on what helps and what hurts. Add to that the intertwined motion and data issues. Certainly, hexapod motion is insufficient, and advanced systems are beyond the reach of even the major airlines. But if we had a sufficiently large validated flight envelope ensuring correct instrument and OTW visual cues, do motion cues improve training transfer, given that they should be ignored? Or do pilots need to be trained to ignore them? Are there effective means to expand the data envelope? Do we know what the exact data requirements are? Is it possible to get accurate models that take into account changes in airplane parameters due to weight, weather, etc.? There is a growing concern that the answer to the last three questions is no.

It is therefore important to convene stakeholders and researchers and coordinate national and international research efforts. A first question would be how to distribute resources between the different approaches to most effectively halt the increase in LOC accidents. Another question is whether the current URT practices help or hurt. Can a convincing argument be made for the feasibility of obtaining valid data? Data that expands the flight envelope so that URT transfers to airplanes with different structural characteristics flown in different configurations in different situations? Follow-up activities to an affirmative conclusion of this endeavor would be 1) development of simulator models and cueing systems that can handle such an envelope and 2) a pilot-in-the-loop validation of the training value of such systems. Concurrently with the development of better training tools would be the development and human-factors evaluation of envelope protections, automated recoveries, and warning and advisory systems. The ultimate concern is to equip initial and recurrent transport-category pilots with lasting skills and effective tools to avoid and recover from losing control of their airplanes.

\section{Acknowledgments}

The authors thank their FAA Program Manager, Dr. Eleana Edens of the Air Traffic Organization Human Factors Research and Engineering Division, and Dr. Thomas Longridge, Manager of the FAA Flight Standards Service Voluntary Safety Programs, for helpful comments and support of this work. We also thank Dr. Stephen Popkin, Chief of the Volpe Center's Human Factors Division; Dr. Michael Zuschlag, an engineering psychologist in the division; and Frank Wang, an aerospace engineer at the Volpe Center, for their pertinent comments.

\section{References}

${ }^{1}$ Civil Aviation Authority (CAA), "Safety Regulation Group Safety Plan 2006," 2006.

2The Boeing Company, "Statistical Summary of Commercial Jet Airplane Accidents: Worldwide Operations 1959-2006," Boeing Commercial Airplanes, 2007.

${ }^{3}$ Klassen, A., "Future Simulation Technologies," World/Regional Aviation Training Conference (WATS/RATS), 2008.

${ }^{4}$ The Boeing Company/Airbus, "Airplane Upset Recovery Training Aid Revision 1," 2004.

${ }^{5}$ Federal Aviation Administration (FAA), "Airworthiness Standards: Transport Category Airplanes," 14 CFR $\S 25.1585$, 2001.

${ }^{6}$ Lacagnina, M., "Cross-Control Upset," Aviation Safety World, 2006, pp. 46-50.

${ }^{7}$ Federal Aviation Administration (FAA), "Flight Simulation Training Device Initial and Continuing Qualification and Use; Final Rule (Change 1)," 14 C.F.R. § 60, 2008.

\footnotetext{
${ }^{8}$ Federal Aviation Administration (FAA), "Low-Altitude Windshear System Equipment Requirements," 14 CFR § 121.358, 1990.

${ }^{9}$ Federal Aviation Administration (FAA), "Appendix E to Part 121 - Flight Training Requirements," 14 CFR § $121,1988$.

${ }^{10}$ Federal Aviation Administration (FAA), "Appendix F to Part 121 - Proficiency Check Requirements," 14 CFR § $121,1977$.
} 
${ }^{11}$ U.S. Department of Transportation, "Report on DOT Significant Rulemakings," 2008.

${ }^{12}$ Federal Aviation Administration (FAA), "Order 8900.1 Flight Standards Information Management System (FSIMS)," 2007.

${ }^{13}$ Higgins, C. R., and Kupics, E. A., "Windshear Training Aid," Federal Aviation Administration/Boeing, 1988.

${ }^{14}$ Fiorino, F., "Delta Upset Training Founded on Simplicity," Aviation Week \& Space Technology, Vol. 157, No. 9, 2002. 2007.

${ }^{15}$ Facsar, F., "Interview mit Lufthansa-Pilot Burkhard Kruse: "Wir hatten Wind mit 300 km/hr"," Spiegel Online, Jan. 19,

${ }^{16}$ International Civil Aviation Organization (ICAO), "Procedures for Air Navigation Services - Training (PANS-TRG)," Draft Doc. 9868, 2006.

${ }^{17}$ Schroeder, C., and Harms, D., "MPL Represents a State-of-the-Art Ab Initio Airline Pilot Training Programme," ICAO Journal, Vol. 62, No. 3, 2007.

18"Point/Counterpoint - The Airbus Position - Extracts," Air Safety Week, Mar. 29, 2004. 994.

${ }^{19}$ National Transportation Safety Board (NTSB), "Safety Study: Commuter Airline Safety," SS--94-02, Washington, DC,

${ }^{20}$ Go, T. H., Bürki-Cohen, J., and Soja, N. N., "The Effect of Simulator Motion on Pilot Vehicle Training and Evaluation," AIAA Modeling and Simulation Technologies Conference, AIAA, 2000.

${ }^{21}$ Go, T. H., Bürki-Cohen, J., Chung, W. W., Schroeder, J., Saillant, G., Jacobs, S., and Longridge, T., "The Effects of Enhanced Hexapod Motion on Airline Pilot Recurrent Training and Evaluation," AIAA Modeling and Simulation Technologies Conference, AIAA, 2003.

${ }^{22}$ Bürki-Cohen, J., and Go, T. H., "The Effect of Simulator Motion Cues on Initial Training of Airline Pilots," AIAA Modeling and Simulation Technologies Conference, AIAA, 2005.

${ }^{23}$ Bürki-Cohen, J., Go, T. H., Chung, W. W., Schroeder, J., Jacobs, S., and Longridge, T., "Simulator Fidelity Requirements for Airline Pilot Training and Evaluation Continued: An Update on Motion Requirements Research," International Symposium on Aviation Psychology (ISAP), 2003.

${ }^{24}$ van der Pal, J., "The Effect of Simulator Motion on Parameter Training for F-16 Pilots," Engineering Psychology and Cognitive Ergonomics: Transportation Systems, Medical Ergonomics and Training, edited by D. Harris, Ashgate, Oxford, England, 1999, pp. 267-275.

${ }^{25}$ Bürki-Cohen, J., Soja, N. N., and Longridge, T., "Simulator Platform Motion--The Need Revisited," International Journal of Aviation Psychology (IJAP), Vol. 8, No. 3, 1998, pp. 293-317.

${ }^{26}$ Bray, R. S., "A Study of Vertical Motion Requirements for Landing Simulation," Human Factors, Vol. 15, No. 6, 1973, pp. 561-568.

${ }^{27}$ Lee, A. T., Flight Simulation: Virtual Environments in Aviation, Ashgate, Burlington, VT, 2005.

${ }^{28}$ Gawron, V. J., "Airplane Upset Training Evaluation Report," Veridian Engineering; National Aeronautics and Space Administration (NASA), NASA/CR 2002-211405, Buffalo, NY, 2002.

${ }^{29}$ Gamauf, M., "Down to the Wire," Business and Commercial Aviation, Vol. 98, No. 5, 2006, pp. 82-85.

${ }^{30}$ McClellan, J. M., "New Gulfstream G650," Flying Magazine, 2008.

${ }^{31}$ Unnikrishnan, S., "Adaptive Envelope Protection Methods for Aircraft," PhD Thesis, School of Aerospace Engineering, Georgia Institute of Technology, Atlanta, 2006.

${ }^{32}$ Air Line Pilots Association (ALPA), "Pilot Authority and Aircraft Protections," 1999.

${ }^{33}$ Hughes, D., "Airbus Shows Interest in Honeywell's Auto Pull-Up Software," Aviation Week, 2005. 
${ }^{34}$ Rosenkrans, W., "Helping Hand," AeroSafety World, Jun. 2008, pp. 17-21.

${ }^{35}$ Wise, J., "Introducing the Airplane of the Future," Popular Mechanics, July 2006.

${ }^{36}$ NASA Research Mission Directorate Aviation Safety Program, "Integrated Resilient Aircraft Control," NASA, (draft research plan), 2007.

${ }^{37}$ Chambers, J. R., "Concept to Reality: Contributions of the NASA Langley Research Center to U.S. Civil Aircraft of the 1990s," NASA, NASA SP-2003-4529, 2003.

${ }^{38}$ Allan, R., "Making the Skies Safer," Electronic Design, May 24, 2004.

${ }^{39}$ Roach, J., "New Icing Detection System for U.S. Airplanes Debuts," National Geographic News, 2006.

${ }^{40}$ Zuschlag, M., and Hayashi, M., "Issues and Knowledge Concerning the Use of Head-Up Displays in Air Transports," USDOT/Volpe National Transportation Systems Center, DOT/FAA/AR-03/50; DOT-VNTSC-FAA-00-05, Cambridge, MA, 2003.

${ }^{41}$ Newman, R. L., and Haworth, L. A., "Flight Displays II: Head-Up and Helmet-Mounted Displays," Spatial Disorientation in Aviation, edited by F.H. Previc and W.R. Ercoline, AIAA, Reston, VA, 2004, Chap. 10, pp. 451-507.

${ }^{42}$ Carbaugh, D., Chen, S., Jacobsen, A., Myers, R., and Wiedemann, J., "Vertical Situation Display for Improved Flight Safety and Reducing Operating Costs," Aero Magazine, No. 20, 2002, pp. 3-11.

${ }^{43}$ George, F., "Flying with Honeywell's Synthetic Vision System," Aviation Week, 2007. 